\title{
Broadband Silica-Based Thulium Doped Fiber Amplifier Employing Dual-Wavelength Pumping
}

\author{
Junjia Wang," Sijing Liang, Yongmin Jung, Qiongyue Kang, Shaif-Ul Alam, and David J. Richardson \\ Optoelectronics Research Centre, University of Southampton, Southampton, SO17 1BJ, UK \\ *jw1g15@soton.ac.uk
}

\begin{abstract}
We report a broadband and gain-flattened silica-based thulium-doped fiber amplifier with dual-wavelength pumping $(790 \mathrm{~nm}+1600 \mathrm{~nm}) .15 \mathrm{~dB}$ gain bandwidth is more than $220 \mathrm{~nm}$ ranging from 1700 to $1920 \mathrm{~nm}$ with a maximum gain of $29 \mathrm{~dB}$ and a noise figure of less than $5 \mathrm{~dB}$. OCIS codes: (060.2320) Fiber optics amplifiers and oscillators; (060.2330) Fiber optics communications.
\end{abstract}

\section{Introduction}

As a result of the growing demand for communication capacity the $2 \mu \mathrm{m}$ wavelength region has been proposed as an attractive new transmission window for optical communications [1]. Thulium doped silica fiber amplifiers (TDFAs) can support amplification over a very broad bandwidth at $2 \mu \mathrm{m}$ and several different TDFA configurations have been presented offering high gain and low noise performance across the wavelength range $1700-2050 \mathrm{~nm}$ [2]. Recently, the short-wavelength amplification edge has been further extended down to $1650 \mathrm{~nm}$ and opens the intriguing possibility of bridging the gap between the long wavelength edge of L-band erbium doped fiber amplifier and short wavelength edge of the TFDA [3]. The wide spectral coverage of TDFAs has attracted much attention in recent years and significant research has focused on further extension of the amplifier coverage. Despite this, it is to be appreciated that the wide spectral coverage of the TDFA has only been realized by concatenating several different TDFA configurations - individual amplifiers can only deliver a limited fraction of the full amplification bandwidth. Typical $15 \mathrm{~dB}$ bandwidths are $\sim 100 \mathrm{~nm}$ at wavelengths around $1750-1800 \mathrm{~nm}$, slightly broader at longer wavelengths around $1900 \mathrm{~nm}$. Here we present a novel broadband TDFA operating in the $1680-1930 \mathrm{~nm}$ region, achieved by employing a dual-wavelength pumping scheme. Dual-wavelength pumping in TDFAs has previously been investigated in the Sband $(1450-1520 \mathrm{~nm})[4,5]$, but no dual-wavelength pumped TDFAs working in $1.7-2 \mu \mathrm{m}$ have been reported so far to the best of our knowledge. The proposed TDFA can extend gain to $<1.7 \mu \mathrm{m}$ whilst providing a much broader amplification window, a lower noise figure (NF) of $<5 \mathrm{~dB}$ and a simpler implementation as compared to the prior art.

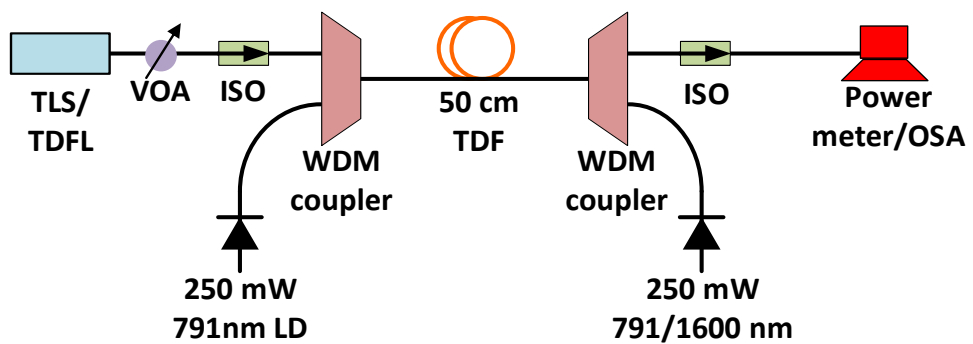

Fig. 1. Schematic of the $791 / 791 \mathrm{~nm}$ LD pumping and $791 / 1600 \mathrm{~nm}$ pumping TDFA. TLS: tunable laser source; TDFL: thulium doped fiber laser; VOA: variable optical attenuator; ISO: isolator; WDM: wavelength division multiplexer; TDF: thulium doped fiber; LD: laser diode.

\section{Experimental setup}

Figure 1 shows a schematic of the experimental setups. We compared two pumping schemes, including $791 / 791 \mathrm{~nm}$ pumping and $791 / 1600 \mathrm{~nm}$ pumping. All configurations were seeded by either a tunable laser source (TLS) provided by Yenista (Tunics T100S-HP) covering the wavelength range $1500-1680 \mathrm{~nm}$, or an in-house built thulium doped fiber laser (TDFL) covering the range $1710-1950 \mathrm{~nm}$. A variable optical attenuator (VOA) was used to adjust the power levels to $-20 \mathrm{dBm}$ and $0 \mathrm{dBm}$ to exhibit small signal and saturated signal scenarios throughout the paper. Isolators were used at both the input and output to suppress parasitic lasing and any unwanted feedback into the seed lasers, thereby improving the stability of the amplifier. The single-mode TDF (OFS TmDF200) has a $\sim 6.5 \mu$ m modefield diameter at $2 \mu \mathrm{m}$ and a core absorption of $\sim 20 \mathrm{~dB} / \mathrm{m}$ at $1.56 \mu \mathrm{m}$. We used a $50 \mathrm{~cm}$ length of TDF in our experiments which was core pumped in a bidirectional configuration by two laser diodes. We used laser diodes operating at $791 \mathrm{~nm}$ (Lumics) delivering up to $250 \mathrm{~mW}$ (24 dBm) of pump power. For $1600 \mathrm{~nm}$ pumping, we used a 
combination of TLS and L-band EDFA delivering $250 \mathrm{~mW}$ average power, as we were short of laser diodes operating at this wavelength. Two different WDM couplers were used to combine the signal and pumps at different wavelengths. An 800 / $1700 \mathrm{~nm}$ fused WDM coupler was used for $791 \mathrm{~nm}$ pumping and a 1550 / $2000 \mathrm{~nm}$ thin-film filter based WDM coupler was used for $1600 \mathrm{~nm}$ pumping. A power meter and an optical spectrum analyzer (Yokogawa AQ6375) were used to measure the gain and NF.

\section{Results and discussion}

Figure 2(a) shows the detailed characterization of both TDFA schemes, i.e. $791 / 791 \mathrm{~nm}$ LD pumping and $791 / 1600$ $\mathrm{nm}$ pumping. The external small signal gain (measured with an input signal power of $-20 \mathrm{dBm}$ ) and external saturated gain (measured with an input signal power of $0 \mathrm{dBm}$ ), along with the external NFs are shown for both pumping schemes. The total pump power launched into the TDF was fixed at $27 \mathrm{dBm}$ in all cases. The $791 / 791 \mathrm{~nm} \mathrm{LD}$ pumping provides a small-signal peak gain of $23 \mathrm{~dB}$ at $1800 \mathrm{~nm}$ and supports $15 \mathrm{~dB}$ gain over a $175 \mathrm{~nm}$ wide window from $1725 \mathrm{~nm}$ to $1900 \mathrm{~nm}$. The spectral gain profile is flat over a $130 \mathrm{~nm}$ wide window spanning from $1740-1870$ $\mathrm{nm}$. The saturated gain has a similar amplification window and varies between $11-14.5 \mathrm{~dB}$ in the $1710-1930 \mathrm{~nm}$ waveband. The external NFs for both small and saturated signals are below $5 \mathrm{~dB}$ from $1710-1860 \mathrm{~nm}$. Note that the $\mathrm{NF}$ increases at longer wavelengths are due to the fact that the insertion loss of our passive components (WDM couplers and isolators) increases at longer wavelength. Compared to $791 / 791 \mathrm{~nm}$ LD pumping, the $791 / 1600 \mathrm{~nm}$ pumping provides a $5 \mathrm{~dB}$ higher peak gain and a $35 \mathrm{~nm}$ broader $15 \mathrm{~dB}$ gain bandwidth spanning from $1700-1920$ $\mathrm{nm}$. There is no significant difference in NFs between the two pumping schemes.
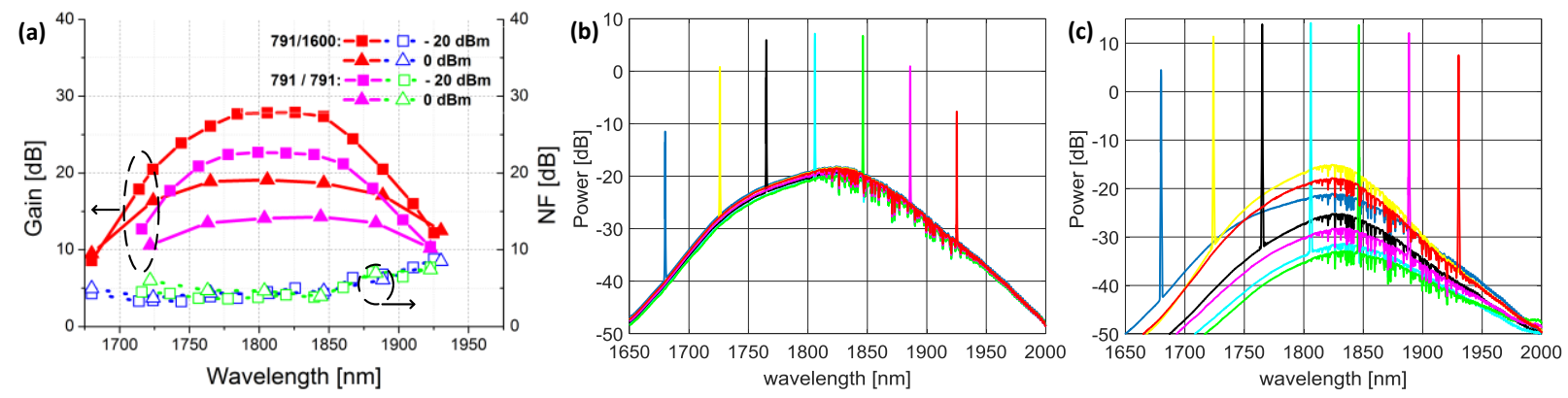

Fig. 2. (a) Detailed broadband performance of the $791 / 791 \mathrm{~nm}$ LD pumping and 791 / $1600 \mathrm{~nm}$ pumping TDFA. 791 / $791 \mathrm{~nm}$ LD pumping: red and blue; 791 / $1600 \mathrm{~nm}$ pumping: pink and green. Amplified (b) small and (c) saturated signals using dual-wavelength pumping. Measured with $0.5 \mathrm{~nm}$ optical spectrum analyzer resolution.

Figure 2(b-c) shows the amplified small and saturated signal spectrum for the $791 / 1600 \mathrm{~nm}$ pumped TDFA. The amplified small signal has $26-30 \mathrm{~dB}$ in-band optical signal-to-noise ratio (OSNR) across the entire amplification band. The amplified saturated signal has $48-50 \mathrm{~dB}$ in-band OSNR and up to $50 \mathrm{~dB}$ out-of-band OSNR.

\section{Conclusions}

We have shown that it is possible to implement a silica based TDFA employing a dual-wavelength pumping, thereby allowing us to obtain a low-noise broadband TDFA covering an amplification window of more than $220 \mathrm{~nm}$ with small signal gain as high as $29 \mathrm{~dB}$ and NF as low as $3.3 \mathrm{~dB}$. The TDFA has a flat-top gain profile and high spectral quality should find use in various communication and sensing applications where optical bandwidth in this operating range is key.

\section{Acknowledgements}

The authors acknowledge David Heard from Yenista for providing the tunable laser source. This work is funded by UK EPSRC through grant EP/I01196X/1 (HYPERHIGHWAY).

\section{References}

[1] M. Petrovich et al., Optics express, vol. 21, pp. 28559-28569, 2013.

[2] Z. Li, et al., Optics Express, vol. 21, pp. 26450-26455, 2013.

[3] Z. Li et al., in Optical Fiber Communication Conference, Los Angeles, California, 2015, p. Tu2C.1.

[4] A. Gomes et al., IEEE Photonics technology letters, vol. 15, pp. 200-202, 2003.

[5] T. Kasamatsu, Y. Yano, and T. Ono, IEEE Photonics Technology Letters, vol. 13, pp. 31-33, 2001. 